Indexed by

\title{
EXPERIMENTAL RESEARCH OF A SMALL-SIZED COMPRESSOR UNIT FOR ABNORMAL OIL AND GAS PRODUCTION CONDITIONS
}

Crossref

KOBSON

8) Google
Yuri Appolonievich Sazonov

National University of Oil and Gas, Gubkin University, Faculty of Mechanical Engineering, Department of Machinery and Equipment for Oil and Gas Industry,

Moscow, Russian Federation

\section{Mikhail Alexandrovich} Frankov

National University of Oil and Gas, Gubkin University, Institute of Petrophysics, Moscow, Russian Federation
Mikhail Albertovich Mokhov

National University of Oil and Gas, Gubkin University, Faculty of Oil and Gas Field Development, Department of Oil Field Development and Operation, Moscow, Russian Federation

Victoria Vasilievna Voronova National University of Oil and Gas, Gubkin University, Institute of Petrophysics,

Moscow, Russian Federation
Petr Vadimovich Pyatibratov National University of Oil and Gas, Gubkin University, Faculty of Oil and Gas Field Development, Department of Oil Field Development and Operation, Moscow, Russian Federation

\section{Inna Vladimirovna Gryaznova} National University of Oil and Gas, Gubkin University, Institute of Petrophysics, Moscow, Russian Federation

Key words: oil production, oil, gas, compressor, multi-phase pump doi:10.5937/jaes18-25999

Cite article:

Sazonov, A. Y., Mokhov, A. M., Pyatibratov, V. P., Frankov, A. M., Voronova, V. V., \& Gryaznova V. I. [2020]. Experimental research of a small-sized compressor unit for abnormal oil and gas production conditions. Journal of Applied Engineering Science, 18(3) 321 - 326. 


\title{
EXPERIMENTAL RESEARCH OF A SMALL-SIZED COMPRESSOR UNIT FOR ABNORMAL OIL AND GAS PRODUCTION CONDITIONS
}

\author{
Yuri Appolonievich Sazonov', Mikhail Albertovich Mokhov², Petr Vadimovich Pyatibratov ${ }^{2}$, \\ Mikhail Alexandrovich Frankov ${ }^{3}$, Victoria Vasilievna Voronova ${ }^{3}$, Inna Vladimirovna Gryaznova ${ }^{3}$ \\ ${ }^{1}$ National University of Oil and Gas, Gubkin University, Faculty of Mechanical Engineering, Department of \\ Machinery and Equipment for Oil and Gas Industry, Moscow, Russian Federation \\ ${ }^{2}$ National University of Oil and Gas, Gubkin University, Faculty of Oil and Gas Field Development, \\ Department of Oil Field Development and Operation, Moscow, Russian Federation \\ ${ }^{3}$ National University of Oil and Gas, Gubkin University, Institute of Petrophysics, Moscow, \\ Russian Federation
}

At the late stage of field development, the application of booster compressor units often becomes an urgent problem. At the same time, this problem remains completely unsolved. Reliable hydraulic machines are required for pumping gas-liquid mixtures with a high content of mechanical impurities. Within the framework of exploratory scientific researches, the practical opportunities to use a group of dynamic type hydraulic machines were assessed. In this respect, the greatest interest from a scientific and practical point of view is small-sized high-speed machinery with the possibility of creating universal multistage pumps and compressors. Labyrinth pumps and compressors, which work more reliably at an increased concentration of mechanical impurities in the flow of the pumped medium, are considered. The new equipment should be characterized by manufacturability, low price and low maintenance costs. As part of the research, new technical solutions are being developed and patented to create a small-sized compressor unit for a wide range of oil and gas production tasks in abnormal operating conditions. The technical problem targeted by the research is to increase the efficiency of the dynamic machine operation in the compressor and multiphase pump modes when pumping gases and gas-liquid mixtures in abnormal operating conditions. New developments can also be focused on improving the efficiency of production and deep processing of raw hydrocarbons, including as it relates to the Arctic environment. It is reasonable to focus further research on optimizing the use of different rotor designs with new materials and new design methods, including additive technologies. The direction of research aimed at increasing the rotor speed is seen as very promising. There is an opportunity to create compact and powerful compressor machinery and pumping equipment.

Key words: oil production, oil, gas, compressor, multi-phase pump

\section{INTRODUCTION}

Oil and gas production in abnormal operating conditions requires efficient pumping and compressor equipment [15], [17]. From the analysis of the state-of-the-art, it can be concluded that the technical problems on the creation of new technologies and equipment for oil and gas production in abnormal operating conditions when pumping gas-liquid mixtures with a large content of mechanical impurities are not yet fully solved. Within the framework of exploratory scientific research, the attempt to estimate opportunities for the practical use of some hydraulic machines of the dynamic type is made. In this respect, the greatest interest from the scientific and practical point of view is small-sized high-speed machinery with the possibilities of creating universal multistage pumps and compressors.

There are known technologies of oil and gas production, where jet pumps are used to pump out the liquid in abnormal operating conditions with a high content of mechanical impurities, as described in patents $[1,2]$ and papers [3-5]. In some cases [6-9], technologies with the pulse mode of gas and liquid flow are recommended. Labyrinth pumps of different designs are used for hydrocarbon production. In comparison with other pumps, labyrinth pumps work more reliably at an increased concentration of mechanical impurities in the liquid flow, as is proven in papers [10-12].

Specialists of Gubkin Russian State University of Oil and Gas (National Research University) conduct research on multiphase pumps of the labyrinth type. Special pumps and turbines have been developed for difficult operational conditions, and each such hydraulic machine is equipped with a rotor made in the form of a mesh. Such rotor design can withstand higher loads, and here we can refer to the experience of the development and application of grid-like wings described in monograph [13]. Some laboratory experiments have shown that this type of machines can be quite easily used as compressor machines for abnormal oil and gas production conditions [18]. It is expedient to develop scientific research in this direction. There is a perspective and technical possibilities for connecting such a compressor to a high-speed gas-turbine engine, at the same time produced and 
pumped gas can be used as fuel [16]. Some tasks on creating vane compressors where the speed of flow can exceed the sound speed can also be considered from new positions.

\section{CONCEPT HEADINGS}

The ongoing research work is focused on the development of new scientific principles for pumping multiphase media and gases with a high content of mechanical impurities in the flow. At the same time, the new pumping technology should be characterized by manufacturability, low price and low maintenance costs. A new scientific approach has been chosen to solve the performance targets. This scientific approach is associated with the creation of new multiphase pumps and compressors using additive technologies. Specialists of Gubkin Russian State University of Oil and Gas (National Research University) conduct scientific research and experimental works to solve urgent problems in the field of hydrocarbons production, including complicated field development conditions. Based on the results of such works, three interrelated research areas for the development of science and technology have been outlined:

1. Pumping technologies and equipment for hydrocarbon production in abnormal operating conditions;

2. Compressor technologies and equipment for hydrocarbon production in abnormal operating conditions;

3. Turbine technologies and equipment, for hydrocarbon production in abnormal operating conditions.

The novelty of scientific and engineering developments is supported by patents in all outlined research areas.

\section{RESULTS}

Within the framework of the research, new technical solutions are being searched for innovative technologies that allow for creating a small-sized compressor unit for a wide range of oil and gas production tasks in abnormal operating conditions.

Within the framework of the patent research, technical solutions for compressors and multiphase pumps have been considered. The multiphase pump according to the patent RU 185434, 2018 contains a housing and a driving shaft with a rotor mounted on it. The housing has input and output channels and grooves in the form of the multiple-start screw thread. The rotor consists of sections installed in sequence on the driving shaft. Each of sections contains a separator installed on the driving shaft and vane wheels, intervane channels of which configured for communication through the grooves of the screw thread in the housing with the wheel intervane channels in the subsequent section. Each subsequent vane wheel in the rotor sections is installed with an angular offset against the previous vane wheel with the formation of multiple-start screw channels. The disadvantage of this well-known technical solution is the relatively narrow speed adjustment range of the rotor because there is a decrease in the efficiency of the working process with increasing rotor speed in the conditions of pumping gas-liquid mixtures. With the rotor speeding-up, the separation process develops more intensively, which leads to a partial separation of the gas-liquid mixture into the liquid fraction and the gas fraction, and then gas starts to accumulate in the central part of the rotor.

The ongoing research is focused on the efficiency improvement of the dynamic machine operation in the compressor mode and the multiphase pump mode when pumping gases and gas-liquid mixtures in abnormal operating conditions.

This problem is solved by the fact that the developed and patented dynamic machine described in the patent [14] contains a housing and a driving shaft with a rotor mounted on it. The housing has input and output channels and grooves in the form of the multiple-start screw thread. The rotor consists of sections installed in sequence on the driving shaft. Each of sections contains a separator installed on the driving shaft and vane wheels, intervane channels of which configured for communication through the grooves of the screw thread in the housing with the wheel intervane channels in the subsequent section. Each subsequent vane wheel in the rotor sections is installed with an angular offset against the previous vane wheel with the formation of multiple-start screw channels. According to the utility model, each section of the rotor is equipped with at least one additional vane wheel with a diameter greater than the diameter of the main vane wheels. The additional vane wheel is located in front of the separator and placed in a cylindrical recess made on the inner housing surface. The cylindrical recess has the depth of the groove, less than the depth of the grooves of the screw thread, made on the inner surface of the housing.

For the convenience of describing the developed technical solution, figures 1-5 present a scheme of a new dynamic machine, considered as the basis for the smallsized compressor unit. Figure 1 shows the isometric scheme of the developed dynamic machine. Figure 2 shows a diagram of a three-section rotor mounted on the driving shaft. Figure 3 shows the housing and Figure 4 shows the longitudinal section of the dynamic machine.

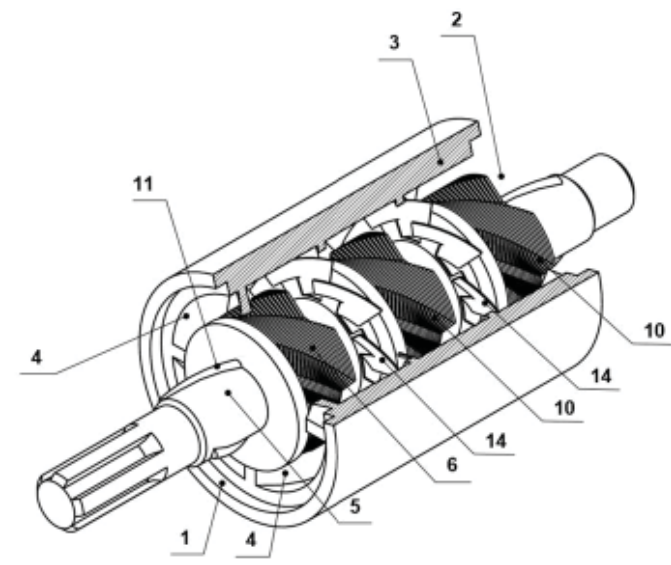

Figure 1: Scheme of the developed dynamic machine 
Figure 5 shows a diagram illustrating the arrangement of the cylindrical recesses made on the inner surface of the housing to place the additional vane wheels with the reduced depth of the screw thread groove, while the arrows show additional vortices in the transition zone of the liquid from the main vane wheels to additional vane wheels with the oversize diameter.

The dynamic machine contains the input 1 and output 2 channels in the housing 3 , which has grooves 4 in the form of the multiple-start screw thread with a depth $\mathrm{H} 1$ of the groove, the driving shaft 5 with the rotor 6 mounted on it. The rotor 6 consists of sections installed in sequence on the driving shaft 5 . There is a spiral groove 7 for the key connection on the driving shaft 5 . Each rotor section contains vane wheels 8 with a diameter $d 1$ mounted on the driving shaft 5 . Between the vanes of the wheel 8 intervane channels 9 , limited by diameter $d 1$ are formed. The intervane channels 9 are configured for communication through the grooves 4 of the screw thread in the housing 3 with the wheel intervane channels 8 in the subsequent section of the rotor. In this example, each section of the rotor contains twenty-two vane wheels 8 , manufactured using the additive technology. Each subsequent vane wheel 8 is mounted with an angular offset against the previous vane wheel 8 , with the formation of multiple-start screw channels 10 in the rotor section. The positioning of each vane wheel 8 on the driving shaft 5 is ensured by a spiral key 11 mounted on the driving shaft 5 in the spiral groove 7 . The groove 7 on the driving

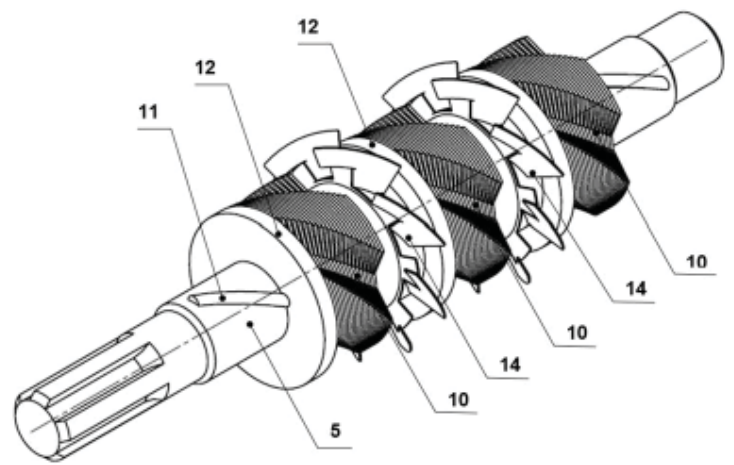

Figure 2: Scheme of the three-section rotor

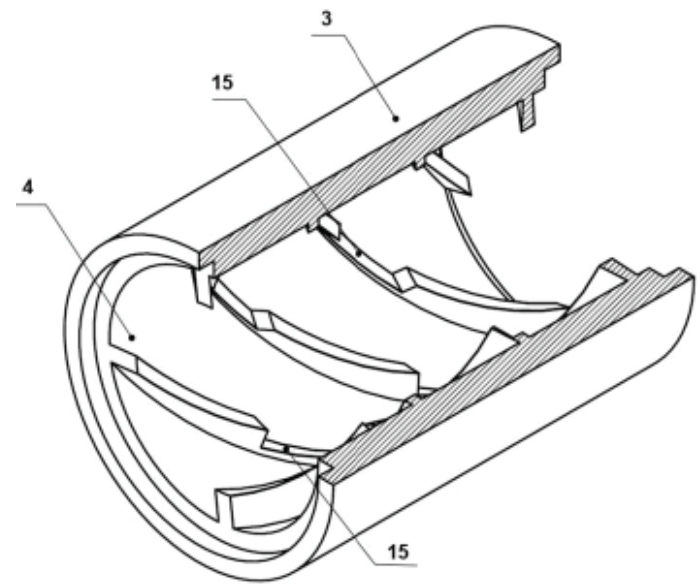

Figure 3: The housing shaft 5 for the spiral key 11 has the screw thread in the opposite direction to the thread in the housing 3 for the spiral grooves 4 . Each section of the rotor contains the separator 12 . The vortices 13 shown by the arrows in figure 5 are generated in front of the separator.

Each section of the rotor is equipped with at least one additional vane wheel with the oversize diameter $d 2$ located in front of the separator 12. Cylindrical recesses 15 are made on the inner surface of the housing 3 for the arrangement of the additional vane wheel 14 with the reduced depth $\mathrm{H} 2$ of the screw thread groove at a section of the cylindrical recess 15 .

The number of additional vane wheels 14 in the rotor sections is selected based on the operating conditions of the machine and taking into account the composition of the pumped medium.

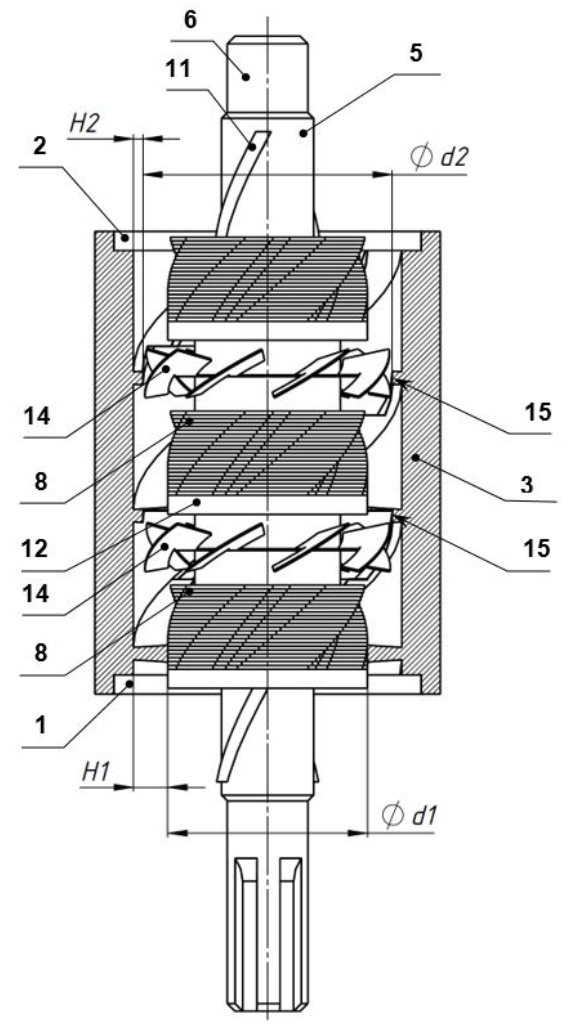

Figure 4: Longitudinal section of the dynamic machine

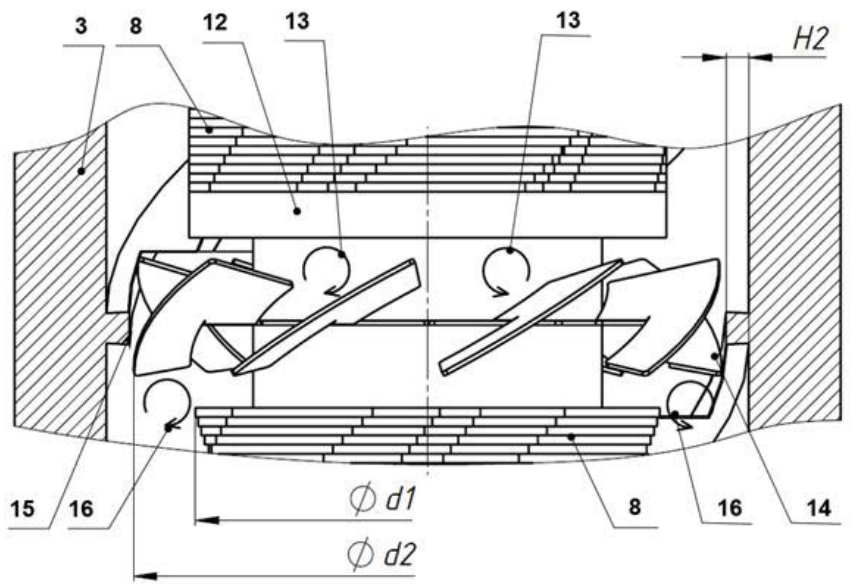

Figure 5: Scheme of the vane wheel arrangement 
The dynamic machine works as follows. When the rotor 6 and, respectively, the vane wheels 8 with the diameter $d 1$ rotate, a force is applied to the pumped gas (or gas-liquid mixture). In this case, the continuous movement of the gas (or gas-liquid mixture) is organized from the intervane channels 9 to the spiral grooves 4 with a depth $\mathrm{H} 1$ of the housing 3 in the direction from the center of the rotor 6 to its periphery. Due to the continuity of the flow, the pumped medium flows continuously into the intervane channels 9 from the spiral grooves 4 . As can be seen from the above, a vortex current is formed in each section of the rotor, which ensures the transfer of energy from each vane wheel 8 to the flow of the pumped medium. The pumped medium with the increased energy is carried away by the vortex flow into the spiral grooves 4 of the housing 3 and is further forced out through the output channel 2. The separator 12 prevents the reverse flow of the gas from the high-pressure area into the low-pressure area. Due to the continuity of the flow through the inlet channel 1 , the gas (or gas-liquid mixture) continuously flows into the pump.

As already pointed out, the positioning of each vane wheel 8 on the driving shaft 5 is ensured by the spiral key 11 mounted on the driving shaft 5 . The groove 7 on the shaft 5 for the spiral key 11 has the opposite screw thread direction against the one in the housing 3 for the spiral key 4.

The separator 12 prevents the transfer of the gas medium from section to section and vortices 13 are formed in front of it, which promote mixing of the medium.

The additional vane wheel 14 with the oversize diameter $d 2$ is located in the housing 3 at the sections of the cylindrical recess 15 with the reduced groove depth of the screw thread $\mathrm{H} 2$. In the flow transition zone from the vane wheels 8 with a diameter $d 1$ to the additional vane wheel 14 with the oversize diameter $d 2$ additional vortices 16 are formed, which mix the gas with the liquid present in the flow, strengthening the process of dispersion of the pumped gas-liquid mixture with the activation of the heat exchange process.

The additional vortices 16 provide strengthening of the dispersion process of the gas-liquid mixture in the multi-

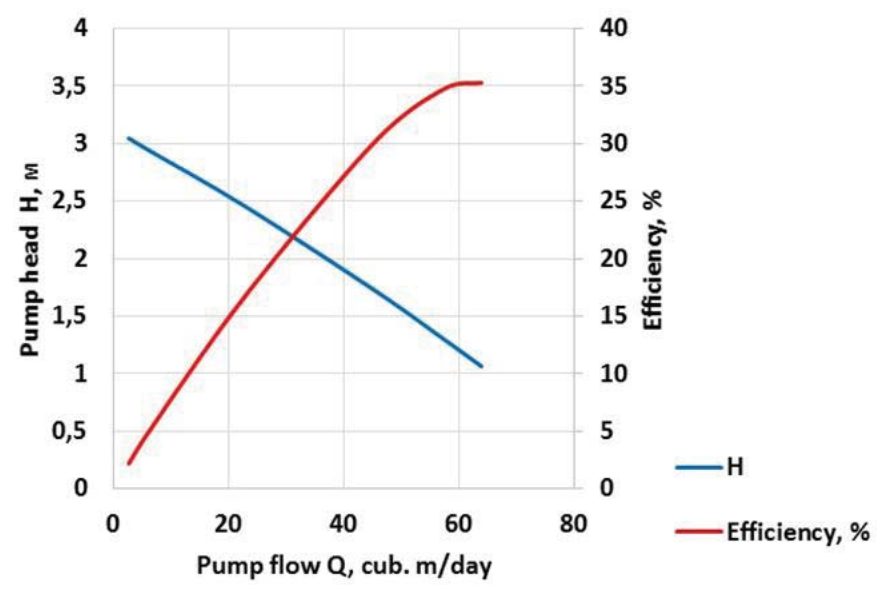

Figure 6: Results of laboratory tests of microlevel models ple-start screw channels 10 in each section of the rotor 6 and at the same time provide weakening of the separation process of the gas-liquid mixture, preventing separation of the gas-liquid mixture into the liquid and gas phases.

With increasing the rotor 6 speed, a proportional and simultaneous increase in the dispersion and separation process speed of the gas-liquid mixture in the cavity of the working chamber is achieved. With increasing the rotor 6 speed, the dispersion process is already developing more intensively, which prevents the partial separation of the gas-liquid mixture into the liquid and gas fractions in the central part of rotor 6 .

The proposed technical solution provides the increased efficiency of the compressor while increasing the rotor speed of the pump in complicated conditions of gas and gas-liquid mixtures pumping. This allows for the expansion of the practical application of new compressors by expanding the adjustment range of the rotor speed.

Tests of micro-level models of labyrinth compressors and pumps were performed under laboratory conditions. Some of these results are shown graphically in Figure 6 . Figure 7 shows a picture of one of the manufactured rotor models within the framework of working out some issues on additive technologies.

The real-world application of the new dynamic machines can be extended when increasing the rotor speed. Pilot tests of created demonstration micro-level models of the compressor have been conducted. The rotor speed was brought up to the level of $20000 . . .30000 \mathrm{rpm}$ in laboratory conditions using a special turbine. The compressed air was used to operate the turbine. Figure 8 shows a photograph of one of the experimental compressors. The compressor housing (orange) can be seen in the photo on the right. The mesh rotor of the special turbine (black) can be seen in the photo on the left.

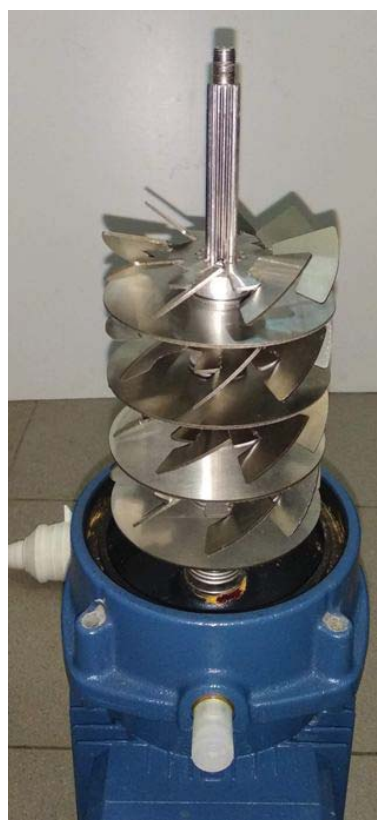

Figure 7: Photograph of the experimental rotor model 


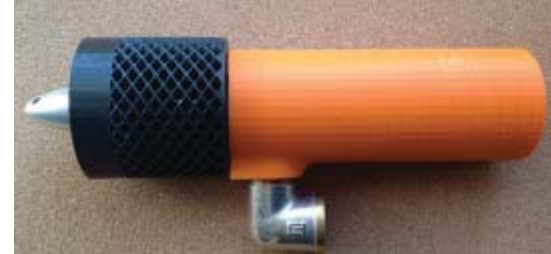

Figure 8: Photograph of the experimental compressor

(the orange compressor housing on the right)

equipped with the experimental turbine

(the turbine black mesh rotor on the left)

Within the framework of scientific research focused on the improvement of dynamic machines, two promising areas of work have been identified. The first area of research, development, testing and engineering is connected with the modernization of the labyrinth compressor for the operation at increased rotor speed. The second one is associated with the use of a new rotor, which has a new geometric shape, different from the known screw. In the course of numerical experiments, the prospective viability of the new labyrinth compressor capable of operating at the increased rotor speed has been shown. Based on the results of the numerical experiments, the demonstration model (micro-level model) of the compressor has been created, and laboratory tests have been conducted. The work capacity of the new labyrinth compressor at the increased rotor speed has been confirmed. The new compressor has a low weight and small overall dimensions. According to preliminary estimates, the mass-topower ratio of the new compressor can be at the level of $0.2 \mathrm{~kg}$ per $1 \mathrm{~kW}$ of power.

\section{CONCLUSIONS}

New scientific principles for pumping gases and multiphase media in the presence of a solid phase in the flow have been developed and patented. The prospects for practical use of new compressors and multiphase pumps have been shown. New methods of hydraulic machinery design have been developed and tested, including the use of additive technologies. When studying the labyrinth pumps, it has been noted that the screw can be replaced by a set of vane wheels. Upon that, the vane wheel can be of enclosed or open type. The vane wheel, as part of the rotor, can be made as a centrifugal wheel or as an axial wheel; other rotor designs are also possible. This area of pumping and compressor technology is still unstudied, taking into account the reversibility of such hydraulic machines when considering the possible turbine mode of operation.

In carrying out scientific research, special dynamic machines have been developed in which the rotor is made in the form of a mesh. Such rotor design is more adapted to high loads, which made it possible to speak about additional possibilities for the creation of more compact and more powerful machines working at higher rotor speed. For the development of this line of research, it is necessary to use the experience gained from the development area of the grid-like wings.
Within the framework of scientific research focused on the improvement of dynamic machines, two promising areas of work have been identified. The first area of research, development, testing and engineering is connected with the modernization of the labyrinth compressor for operation at increased rotor speed. The second one is associated with the use of a new rotor, which has a new geometric shape, different from the known screw. In the course of numerical experiments, the prospective viability of the new labyrinth compressor capable of operating at the increased rotor speed has been shown. Based on the results of the numerical experiments, a demonstration model (micro-level model) of the compressor has been created, and laboratory tests have been conducted. The work capacity of the new labyrinth compressor at the increased rotor speed has been confirmed. The new compressor has a low weight and small overall dimensions. According to preliminary estimates, the mass-to-power ratio of the new compressor can be at the level of $0.2 \mathrm{~kg}$ per $1 \mathrm{~kW}$ of power.

New developments can be focused on improving the efficiency of production and deep processing of raw hydrocarbons, including as it relates to the Arctic environment. Further research should be directed to solving optimization tasks using different rotor designs, new materials and new design methods. The direction of research aimed at increasing the rotor speed is seen as very promising. There is an opportunity to create compact and powerful compressor machinery and pumping equipment.

As can be seen from the above, the development of labyrinth dynamic machines can provide additional impetus to the development of works on hydraulic and gas turbines, as well as on thermal machines where turbine and compressor technology is used.

Some specific findings of the research performed can be used to create new pumping, compressor and turbine equipment for various industries, including transport and robotics industry.

\section{ACKNOWLEDGEMENTS}

The work was performed with the financial support of the Ministry of Education and Science of the Russian Federation within the framework of the state contract in the sphere of scientific activities, topic number FSZE-20200006.

\section{REFERENCES}

1. Falk, K.L. (2014). US Patent $8,863,827$ B2. Jet pump for use with a multi-string tubing system and method of using the same for well clean out and testing. Date of Patent: Oct. 21, 2014.

2. Misselbrook, J. G. (2007). US Patent Application Publication 2007/0187111 A1. Apparatus and method for dewatering low pressure gradient gas wells. Publication Date: Aug. 16, 2007. 
3. Drozdov, A.N. (2011). Research of the pump characteristics at pumping of the gas-liquid mixtures and application of the obtained results for the development of the water-alternated-gas injection technologies. Oil Industry, 9, 108-111.

4. Drozdov, A. N., Terikov, B. A. (2009). Application of the downhole hydro-jet pumps with the double-row lift for the operation of the difficult wells. Oil Industry, $6,68-72$.

5. Drozdov, A. N., Egorov, Yu. A., Telkov, V. P., Verbitsky, V. S., Dengaev, A. V. (2006). Technology and technique of water-alternated-gas injection on oil formations. Oil and Gas Territory, 2, 54-59.

6. Podvidz, L. G. (1980). Pulse-action pumping plants. Proceedings of the Higher Educational Institutions (Izvestiya VUZOV). Machine Building, 9, 51-56.

7. Castel, Y. (1996). US Patent No. 5,575,625. Multiphase pump with sequential jets. Date of Patent: Nov. 19, 1996.

8. Castel, Y. (1997). US Patent No. 5,616,006. Pumping method and device with sequential jets. Date of Patent: Apr. 1, 1997.

9. Castel, Y. (1998). US Patent No. 5,716,196. Pumping method and device with sequential jets. Date of Patent: Feb. 10, 1998.

10. Mokhov, M. A., Sazonov, Yu. A., Frankov, M. A., Tumanyan, Kh. A., Kruglov, S. V. (2019). Development and Research of a Multi-Phase Pump for Oil and Gas Production at a High Content of Mechanical Impurities in the Flow. Journal of Computational and Theoretical Nanoscience, 16(7), 2815-2821(7).

11. Mokhov, M. A., Sazonov, Yu. A., Frankov, M. A., Tumanyan, Kh. A., Kruglov, S. V., Muradov, A. V. (2019). Development and Research of Multi-Phase Reversible Pump. Journal of Computational and Theoretical Nanoscience, 16(7), 3007-3012(6).
12. Mokhov, M. A., Sazonov, Yu. A., Mulenko, V. V., Frankov, M. A., Tumanyan, Kh. A., Timoshenko, V. G., Kruglov, S. V. (2019). Development of Pumping Equipment for Oil and Gas Production in Complicated Conditions. Journal of Computational and Theoretical Nanoscience, 16(11), 4573-4578.

13. Bilotserkovskiy, S. M., Odnovol, L. A., Safin, Yu. Z., Tyulenev, A. I., Frolov, V. P., Shitov, V. A. (1985). Grid-like wings. Moscow: Mechanical Engineering.

14. Sazonov, Yu. A., Mokhov, M. A., Frankov, M. A., Tumanyan, Kh. A. (2019). RU Patent No. 195,298. The pump. Application No. 2019/137559, Application Date: Nov. 22, 2019. Publication Date: Jan. 23, 2020, Bulletin No. 3.

15. Liu, Z., Li, L., Han, Z., Pan, J., Ding, Y. (2016). Current Following Segmented PID Control of Air Supply System in Heavy-Duty PEMFC System. Journal of Southwest Jiaotong University, 51(3).

16. Zheng, S., Yang, L. (2017). Numerical Experiments of Dynamic Response of Buried Gas Pipeline under the Action of Seismic Waves Induced by Tunnel Blasting. Journal of Southwest Jiaotong University, 52(2).

17. Taras'yants, S. A., Pashkov, P. V., Efimov, D. S. (2018). Laboratory Tests of a Jet Device to Increase the Centrifugal and Axial Pump Suction Head. Periodico Tche Quimica, 15 (Special Issue - 1), 55-66.

18. Konesev, S. G., Khlyupin, P. A., Greb, A. V., Kondratiev, E. Y. (2018). Induction Technology in High-Viscosity Oil Production at Tazovskoye Field. Periodico Tche Quimica, 15 (30), 520-526. 